\title{
THE ASYMPTOTIC LOCATION OF THE ROOTS OF A CERTAIN TRANSCENDENTAL EQUATION*
}

BY

\author{
RUDOLPH E. LANGER
}

The determination of the characteristic values in many boundary problems associated with differential or integral equations depends upon the location of the roots of an equation of the form

$$
\left|\phi_{i j}(\lambda)+\psi_{i j}(\lambda) e^{\lambda A_{i}}\right|=0 .
$$

The symbol $\left|\alpha_{i j}\right|$ is used here to designate the determinant of order $n$ with general element $\alpha_{i j}$. In expanded form the equation (1) may be written

$$
\sum \omega_{l}(\lambda) e^{\lambda B_{l}}=0,
$$

where each $B_{l}$ is a combination of the values $A_{j}$, and $\omega_{l}$ is expressible in terms of the coefficients $\phi_{i j}, \psi_{i j}$.

The following are the hypotheses to be made concerning equation (1):

(i) the exponents $A_{j}$ are complex constants;

(ii) the functions $\phi_{i j}(\lambda), \psi_{i j}(\lambda)$ are such that the coefficients $\omega_{l}(\lambda)$ of the equation in the expanded form (2) are analytic for $|\lambda|$ sufficiently large, and are of the form

$$
\omega_{l}(\lambda)=\lambda^{\nu_{l}}\left[a_{l}\right],
$$

where $\nu_{l}$ is real and $a_{l}$ is a constant not zero. If $\nu$ is not an integer, $\lambda^{v}$ is taken to mean $\exp (\nu \log \lambda)$, the principal value of the logarithm to be chosen.

The symbol $\left[a_{l}\right]$ in (3) designates a quantity $a_{l}+\epsilon(\lambda)$, where $\epsilon(\lambda)$ is used as a generic designation for a function which is analytic for $|\lambda|$ sufficiently large and which approaches zero uniformly as $|\lambda| \rightarrow \infty$.

The equation of the type (1) either in form (1) or form (2) has been extensively studied under hypotheses of varying degrees of restrictiveness, by Tamarkin, $\dagger$ Wilder, $\ddagger$ and Pólya and Schwengeler. $\S$ Of these, the two last

\footnotetext{
* Presented to the Society, March 30, 1929. Received by the editor of the Bulletin in March 1929, accepted for publication in the Bulletin, and subsequently transferred to these Transactions.

$\dagger$ (1) Some General Problems of the Theory of Ordinary Linear Differential Equations etc., Petrograd, 1917 (in Russian), also (2), Mathematische Zeitschrift, vol. 27 (1927), p. 24.

$\ddagger$ These Transactions, vol. 18 (1917), p. 415.

$\S$ Pólya, Sitzungsberichte der Bayerischen Akademie, München, 1920, p. 285.

Schwengeler, E., Geometrisches über die Verteilung der Nullstellen etc., Zürich, 1925.
} 
named focus the attention in large measure upon the geometric determination of the configuration of regions in which the roots of the equation are located. They consider the case in which the coefficients are polynomials; a restriction which would appear from the following discussion to be unessential. The present note is intended as a brief analytic rather than geometric discussion. The final result is more precise than that obtained by Schwengeler. ${ }^{*}$ The deductions are based directly on the work of the other authors cited above. This work thus appears, indeed, to include in large measure the analytic deductions on the location of the roots made subsequently by Schwengeler.

The constants $A_{j}$ are by hypothesis complex. We designate by $\mu_{1}, \mu_{2}$, $\cdots, \mu_{r}$ the distinct values of the set $\left(\arg A_{j}\right)$ arranged in the order

$$
0 \leqq \mu_{1}<\mu_{2}<\cdots<\mu_{r}<\pi .
$$

The restriction of the values $\mu_{j}$ to the interval $(0, \pi)$ is unessential, inasmuch as the multiplication of the $j$ th column of equation (1) by $\exp \left(-\lambda A_{j}\right)$ leaves the type of the equation unchanged and substitutes $\left(-A_{j}\right)$ in the rôle of $A_{j}$. We redesignate now by $A_{h k}, k=1,2, \cdots$, those of the quantities $A_{j}$ with argument $\mu_{h}$, arranging them in the order

The rays

$$
\left|A_{h k}\right|<\left|A_{h, k+1}\right| \text {. }
$$

$$
\arg \lambda=\frac{\pi}{2}-\mu_{h}
$$

proceed with $h$ in clockwise succession in the $\lambda$ plane, and are followed in this succession by the rays

$$
\arg \lambda=-\frac{\pi}{2}-\mu_{h}
$$

We shall confine the attention to a sector $S_{c}$ enclosing a single one of the rays $(4),\left(4^{\prime}\right)$, i.e.

$$
\arg \lambda= \pm \frac{\pi}{2}-\mu_{c}
$$

and defined by the relations

$$
S_{c}: \pm \pi / 2-\mu_{c}+\eta_{c} \geqq \arg \lambda> \pm \pi / 2-\mu_{c+1}+\eta_{c+1},
$$

where $\eta_{c}, \eta_{c+1}$ are positive constants chosen sufficiently small but otherwise arbitrary. The character of equation (1) in sector $S_{c}$ is typical of its character

\footnotetext{
* Cf. Schwengeler, loc. cit., p. 48, and the final theorem of this note.
} 
in any analogous sector associated with another of the rays (4) or $\left(4^{\prime}\right)$. The deductions for sector $S_{c}$, therefore, reveal the facts for the entire $\lambda$ plane.

If the determinant in equation (1) is expanded the equation may be written in the form

$$
\sum_{l=0}^{L} e^{\lambda K_{l}} \sum_{p=1}^{P} \omega_{l_{p}}(\lambda) e^{\lambda K_{p}^{\prime}}=0,
$$

in which the constants $K_{p}^{\prime}$ are linear combinations with coefficients 0 and 1 of the quantities $A_{h k}, h \neq c$, and the constants $K_{l}$ are similar combinations of the quantities $A_{c k}$. We may choose the subscripts, moreover, so that

$$
0=K_{0}<\left|K_{1}\right|<\cdots<\left|K_{L}\right| \text {. }
$$

Inasmuch as the quantities $R\left(\lambda A_{h k}\right),{ }^{*}$ with $h \neq c$, maintain their signs throughout the sector $S_{c}$, the order by numerical magnitude of the quantities $R\left(\lambda K_{p}^{\prime}\right)$ is fixed for $\lambda$ in the sector. If we suppose then that the subscript 1 is assigned so that

$$
R\left(\lambda K_{1}^{\prime}\right) \geqq R\left(\lambda K_{p}^{\prime}\right)
$$$$
(p=2, \cdots, P)
$$

and divide equation (6) by $\exp \left(\lambda K_{1}^{\prime}\right)$ we have

$$
\sum_{l=0}^{L} e^{\lambda K_{l}}\left\{\omega_{l}(\lambda)+\sum_{p=2}^{P} \omega_{l p}(\lambda) e^{\lambda B_{p}}\right\}=0,
$$

where $\omega_{l}=\omega_{l_{1}}$, and $H_{p}=K_{p}^{\prime}-K_{1}^{\prime}$. In $S_{c}$ we have then

$$
R\left(\lambda H_{p}\right)<0 .
$$

Let $l^{\prime}$ and $l^{\prime \prime}$ be defined now by the relations

$$
\begin{gathered}
\omega_{l} \equiv 0 \text { for } l<l^{\prime}, \text { and } l>l^{\prime \prime}, \\
\omega_{l^{\prime}} \neq \equiv 0, \quad \omega_{l^{\prime \prime}} \not \equiv 0, \dagger
\end{gathered}
$$

and let the sector $S_{c}$ be divided into sub-sectors $S_{c 1}$ and $S_{c 2}$ as follows:

$$
\begin{aligned}
& S_{c 1}: \pm \pi / 2-\mu_{c}+\eta_{c} \geqq \arg \lambda> \pm \pi / 2-\mu_{c}-\eta_{c}, \\
& S_{c 2}: \pm \pi / 2-\mu_{c}-\eta_{c} \geqq \arg \lambda> \pm \pi / 2-\mu_{c+1}+\eta_{c+1} .
\end{aligned}
$$

We observe then readily that if $\eta_{c}$ is chosen sufficiently small we have in $S_{c 1}$ because of (8)

$$
\left\{\omega_{l_{p}} e^{\lambda H_{p}}\right\} \exp \left(\lambda K_{l}\right)=\omega_{l_{1}}[0] \exp \left(\lambda K_{l_{1}}\right)(p=2, \cdots, P),
$$

* The symbol $R(x)$ designates "the real part of $x$. .

$\dagger$ In the simplest case we should have $l^{\prime}=0, l^{\prime \prime}=L$. 
for any values of $l$ and $l_{1}$. Equation (7) may be written, therefore, in the form

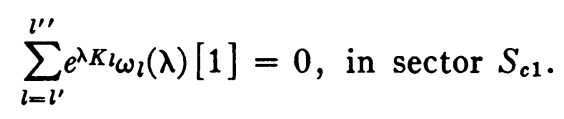

In the sector $S_{\mathrm{c} 2}$ on the other hand relations (9) are valid only for $l \leqq l_{1}$. We have, however, further in this case

$$
\omega_{l} \exp \left(\lambda K_{l}\right)=\omega_{l_{1}}[0] \exp \left(\lambda K_{l_{1}}\right), \text { for } l<l_{1},
$$

and hence we may write the equation after division by $\exp \left(\lambda K_{l^{\prime \prime}}\right)$ in the form

$$
\omega_{l^{\prime \prime}}(\lambda)[1]+\sum_{l=l^{\prime \prime}}^{L} \sum_{p=2}^{P} \omega_{l_{p}}(\lambda)[1] \exp \left(\lambda\left\{K_{l}-K_{l^{\prime \prime}}+H_{p}\right\}\right)=0 .
$$

If $l^{\prime \prime}=L$ the left member of this consists of its first term alone, and because of (3) there will be no roots of the equation in $S_{c 2}$ for $|\lambda|$ sufficiently large. On the other hand if $l^{\prime \prime}<L$ the equation (11) is of precisely the original type (6) and we may repeat the foregoing discussion. The sector $S_{c}$ and the ray (5) must be replaced in their rôles by the sector $S_{c 2}$ and the ray

$$
\arg \lambda= \pm \pi / 2-\mu_{c 2},
$$

where $\mu_{c 2}$ is the largest value of the set

$$
\arg \left\{K_{l}-K_{l^{\prime \prime}}+H_{p}\right\}, l>l^{\prime \prime},
$$

and the quantities $\lambda K_{l}$ must be replaced by those of the quantities $\lambda\left\{K_{l}-K_{l \prime}\right.$, $\left.+H_{p}\right\}$ whose real part vanishes on the ray (12). We find from a repetition of the argument then that in a sector enclosing the ray (12) the equation is again of the general form (10), and that the remaining part of $S_{c 2}$ is either devoid of roots for $|\lambda|$ sufficiently large, or else that a further repetition of the procedure leads to a third ray to be considered. In any event the number of rays thus found in $S_{c}$ and hence in the entire plane is finite. Every such ray is determined by a relation

$$
R(\lambda Q)=0,
$$

where $Q$ is formed by linear combination with coefficients 0,1 , and -1 , from the values $A_{j}$ of equation (1), and in an arbitrarily small sector enclosing such a ray the equation is of type (10), i.e. in virtue of (3) of the form

$$
\sum_{m=0}^{M} \lambda^{v_{m}}\left[\alpha_{m}\right] e^{\lambda Q_{m}}=0 .
$$

Excepting in these small sectors the equation has no roots for $|\lambda|$ sufficiently large. 
The location of the roots of the equation (14) remains to be determined, and to this end we quote the following theorems which deal with the equation in certain specialized forms.

TheOREM A.* If $B$ and $K$ are real, $B>0$, then for $|\rho|$ sufficiently large the roots $\rho_{m}$ of the equation

$$
\left[a_{1}\right]+\rho^{K}\left[a_{2}\right] e^{\rho B}=0
$$

are asymptotically spaced along the curve

and

$$
\left|\rho^{K}\left(\frac{a_{2}}{a_{1}}\right) e^{\rho B}\right|=1,
$$

$$
\left|\rho_{m}\right| \sim B^{-1}\left\{ \pm\left(2 m-\frac{K}{2}\right) \pi+\arg \left(-\frac{a_{2}}{a_{1}}\right)\right\} .
$$

Moreover if $\rho$ remains uniformly away from the roots of (15) then the left hand member of the equation is uniformly bounded from zero.

We note that for $|\rho|$ sufficiently large one branch of the curve (16) lies entirely within an arbitrarily small sector about the positive axis of imaginaries and approaches parallelism with this axis, while the other branch is similarly located with respect to the negative axis of imaginaries.

THEOREM B. $\dagger$ If the constants $B_{j}$ are real and

$$
0=B_{0}<B_{1}<\cdots<B_{J},
$$

then for $|\rho|$ sufficiently large the roots of the equation

$$
\sum_{j=0}^{J}\left[b_{j}\right] e^{\rho B_{i}}=0, \quad b_{0} \neq 0, \quad b_{J} \neq 0,
$$

lie in the strip bounded by the lines

$$
R(\rho)= \pm c
$$

where $c$ is a suitably chosen real constant. The number $N$ of roots $\ddagger$ lying in any interval of this strip of length $l$ satisfies the relation

$$
B_{J} t /(2 \pi)-(J+1) \leqq N \leqq B_{J} l /(2 \pi)+(J+1) .
$$

Moreover if $\rho$ remains uniformly away from the zeros of the equation (17) the left hand member of the equation is uniformly bounded from zero.

* Wilder, loc. cit., pp. 424, 425. Tamarkin, loc. cit. (1), pp. 284-289. This theorem and Theorem $\mathrm{B}$ are proved by the authors cited under the hypotheses that $K$ is an integer and that $[a]=a+E(\rho) / \rho$, where $E(\rho)$ is bounded for $|\rho|$ sufficiently large. Their proofs, however, are still valid with the more general hypotheses of this paper.

† Wilder, loc. cit., p. 421-423. Tamarkin, loc. cit. (1), pp. 168-173, (2), pp. 27-29.

$\S$ Counted with their multiplicities. 
We consider now the transformation of this theorem by means of the substitution

$$
\rho=\mu+\sigma \log \mu,
$$

in which $\sigma$ is a real constant and the principal value of the logarithm is to be understood. The region into which (18) is transformed is bounded by the curves

$$
\left|\mu^{\sigma} e^{\mu}\right|=e^{ \pm c} .
$$

It is readily shown to be a strip asymptotically of constant width $2 c$, and, like the curve (16), approaching parallelism with the axes of imaginaries.

If we designate by $\rho^{\prime}$ and $\rho^{\prime \prime}$ the real and imaginary parts of $\rho$, with corresponding notation for $\mu$, the relation (19) may be resolved into the equalities

$$
\begin{aligned}
& \rho^{\prime}=\mu^{\prime}+\sigma \log |\mu|, \\
& \rho^{\prime \prime}=\mu^{\prime \prime}+\sigma \arg \mu .
\end{aligned}
$$

From the first of these we obtain, upon dividing it by $|\mu|$, restricting $\rho$ to the region (18), and allowing $|\mu|$ to increase indefinitely, the result $\mu^{\prime} /|\mu| \rightarrow 0$, i.e. $\arg \mu \rightarrow \pm \pi / 2$. It follows readily that the portion $T_{R, l}$ of region (20) which lies between the arcs $|\mu|=R$, and $|\mu|=R+l$, is asymptotically congruent, as $R \rightarrow \infty$ with $l$ fixed, to the corresponding portion of strip (18), and this in turn approaches conformity with a piece of the strip of length $l$. Moreover if $\mu_{1}$ and $\mu_{2}$ lie in $T_{R, l}$ and correspond to $\rho_{1}$ and $\rho_{2}$, then clearly

$$
\left(\rho_{2}-\rho_{1}\right)-\left(\mu_{2}-\mu_{1}\right) \rightarrow 0, \quad \text { as } \quad R \rightarrow \infty .
$$

Hence when $R$ is sufficiently large any two points of $T_{R, l}$ at a distance greater than $\delta$ from each other correspond to points of strip (18) which are at a distance greater than $\frac{1}{2} \delta$ from each other. Inasmuch as a function $\epsilon(\rho)$ clearly becomes a function $\epsilon(\mu)$ we may state the following theorem in which we have again replaced the $\mu$ of the preceding discussion by $\rho$.

Theorem C. If $0=B_{0}<B_{1}<\cdots<B_{J}$, and $\sigma$ is real, then for $|\rho|$ sufficiently large the roots of the equation

$$
\sum_{j=0}^{J}\left[b_{j}\right]\left\{\rho^{\sigma} e^{\rho}\right\}^{B_{i}}=0, \quad b_{0} \neq 0, \quad b_{J} \neq 0,
$$

lie within the strip bounded by a pair of curves (20). The number $N$ of roots 
lying in a portion of this strip cut out by a pair of arcs $|\rho|=R$, and $|\rho|=R+l$, where $l$ is fixed, satisfies when $R$ is sufficiently large the relation

$$
\frac{B_{J} l}{2 \pi}-(J+1) \leqq N \leqq \frac{B_{J} l}{2 \pi}+(J+1) .
$$

Moreover if $\rho$ remains uniformly away from the zeros of (21) then the left hand member of the equation is uniformly bounded from zero.

Consider now the equation of type (14), i.e.

$$
\sum_{m=0}^{M} \rho^{\nu_{m}}\left[a_{m}\right] e^{\rho C_{m}}=0,
$$

in which the constants $C_{m}$ are real and $0=C_{0}<C_{1}<\cdots<C_{m}$. Since multiplication of the equation by any power of $\rho$ is permissible we may suppose $\nu_{0}=0$. Let $\sigma_{1}$ and $m_{1}$ be determined by the relations

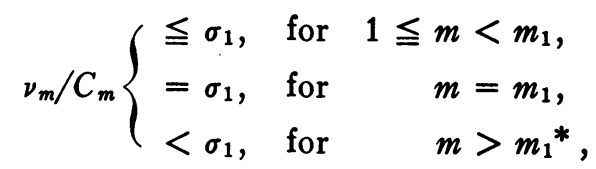

and choose $\eta_{1}$, so that for every $m \geqq 1$

either

$$
\begin{gathered}
\nu_{m} / C_{m}=\sigma_{1} \\
\nu_{m} / C_{m}<\sigma_{1}-\eta_{1} .
\end{gathered}
$$$$
\text { or }
$$

Further let $S_{1}$ designate the region bounded on the left by any ray through the origin with argument between $\pi / 2$ and $\pi$, and on the right by the curve

$$
\left|\rho^{\sigma_{1}} e^{\rho}\right|=e^{\boldsymbol{v}_{1}} \text {. }
$$

In the region $S_{1}$ we have

$$
\rho^{\nu_{m}} e^{\rho C_{m}}=\left\{\begin{array}{ccc}
\left\{\rho^{\sigma_{1}} e^{\rho}\right\}^{C_{m}}, & \text { if } & \sigma_{1}=\nu_{m} / C_{m}, \\
{[0],} & \text { if } & \sigma_{1}>\nu_{m} / C_{m} .
\end{array}\right.
$$

Hence in $S_{1}$ the equation (22) is of the form (15) or (21) and its roots are located as described in Theorem A or Theorem $\mathrm{C}$.

On the other hand in any region $S_{2}$ bounded on the left by the curve (23) we have

$$
\rho^{\nu_{m}} e^{\rho C_{m}}=\left\{\rho^{\sigma_{1}} e^{\rho}\right\} C_{m}[0], \text { for } m<m_{1} .
$$

Hence equation (22) is of the form

\footnotetext{
* In the simplest case we should have $m_{1}=M$.
} 


$$
\sum_{m=m_{1}}^{M} \rho^{\eta_{m^{\prime}}}\left[a_{m}\right] e^{\rho C_{m^{\prime}}}=0, \quad \text { in } S_{2},
$$

where $\nu_{m}^{\prime}=\nu_{m}-\nu_{m_{1}}$, and $C_{m}^{\prime}=C_{m}-C_{m_{1}}$. If $m_{1}=M$ this latter equation has no roots for $|\rho|$ large. On the other hand if $m_{1}<M$ the equation is precisely of the type (22) and the discussion applied above to $S_{1}$ may be repeated for $S_{2}$. The roots of equation (22) are thus found to be confined to a finite number of strips of the type (20).

Lastly to apply to equation (14) the results obtained for equation (22) it is necessary only to set

$$
\lambda=\rho e^{-i \arg Q_{m}} .
$$

In conclusion we state, therefore, the following theorem.

THEOREM. For $|\lambda|$ sufficiently large the roots of equation (1) lie within arbitrarily small sectors each containing a ray (13). Within each of these sectors the roots are further confined to a finite number of strips which are asymptotically of constant width and approach parallelism with the ray (13) enclosed in the sector. The number of roots $N$, counted with their multiplicities, which lie in any portion of such a strip bounded by a pair of arcs $|\lambda|=R$, and $|\lambda|=R+l$, satisfies, for $R$ sufficiently large, a relation

$$
\alpha l-\beta \leqq N \leqq \alpha l+\beta,
$$

in which $\alpha$ and $\beta$ are suitably determined constants. Moreover if $\lambda$ remains uniformly away from a root of equation (1) the left hand member of the equation is uniformly bounded from zero.

UNIVERSITY OF WISCONSIN, Madison, Wis. 